\title{
Health Education Intervention on Knowledge and Accessibility of Pregnant Women to Antenatal Care Services in Edu, Kwara State, Nigeria
}

\author{
Umar N. Jibril ${ }^{*}$, Garba N. Saleh ${ }^{2}$, Olusegun Badaki ${ }^{3}$, Emmanuel E. Anyebe $^{4}$, Aliyu Umar $^{5}$, Abdukadir \\ Kamal $^{6}$
}

\begin{abstract}
Objectives: Poor knowledge about importance of early antenatal care visit and awareness on health risk during pregnancy among pregnant women prevented them from accessing early antenatal care services. This study examined the impact of health education intervention (HEI) on access to antenatal care services by pregnant women in Edu Local Government Area (LGA), Nigeria.

Materials and Methods: A quasi-experimental research employing pre-test and post-test control group design was adopted. A researchers-designed questionnaire was used for data collection and the split-half test of reliability was used to determine its reliability. One hundred and twenty pregnant women were exposed to 10 weeks of HEI after pre-test, 60 experimental groups were exposed to the lectures on antenatal care services, and 60 control groups were exposed to HIV/AIDS sessions, followed by post-test. The descriptive statistics was used to answer research questions, while the inferential statistics of $\mathrm{t}$-test was used to test the hypothesis at 0.05 significance level.

Results: This study showed upward shift from pre-intervention mean of 71.08 to 93.33 post intervention mean in the experimental groups which implied the positive impact of HEI on the knowledge and willingness of pregnant women to access antenatal care services in Edu LGA.

Conclusions: It was concluded that HEI could have positive impact on the knowledge and willingness of pregnant women to access antenatal care services, hence HEI needs to be sustained to improve the pregnant women's health-seeking behaviors about antenatal care services in Edu LGA.

Keywords: Antenatal Care Services, Community-Based Intervention, Health Education Intervention, Knowledge and accessibility, pregnant women
\end{abstract}

\section{Introduction}

Pregnant women, in the age range of 15-49, who constitute more than one-fifth of the world's population, are repeatedly exposed to pregnancy and childbearing risk (1). Antenatal care (ANC) is a precise and systematic assessment and follow-up care including education, counseling, screening, and treatment that is provided for pregnant women to assure the best possible health of a mother and her fetus (2). Likewise, ANC refers to the prenatal health care which is provided for pregnant women and their children during pregnancy by the skilled health personnel. The World Health Organization (WHO) recommended that pregnant women are expected to visit the antenatal clinic 4-5 times before delivery for any medical problems; three antenatal visits are at least recommended, ideally with the first visit early in the pregnancy. This number may vary according to national and institutional policies (3). In Nigeria, four
ANC visits are recommended for every pregnant woman before delivery (4). The primary objective of ANC visit is to establish contact with the women, to identify and manage current as well as potential risks and problems, and to improve maternal health which is one of the eightmillennium development goals (MDGs). Under MDG5, countries committed to reduce maternal mortality by three-quarters between 1990 and 2015. Since 1990, maternal deaths worldwide have dropped by $47 \%(4,5)$.

Women's knowledge and attitude regarding health and medical care must be investigated, during pregnancy and childbirth, to identify any suspicion or fear of medical interventions such as hospital delivery, caesarean section, or blood transfusion among women and to ascertain whether appropriate information, education, and communication (IEC) activities are required (6). Health knowledge is considered as one of the key factors in saving 
the women's lives from preventable causes of maternal death in that it enables women to be aware of their rights and to seek for appropriate health care services. Crucial information is needed for most effective and efficient treatment to be organized; this information consists of whether women are aware of the riskful signs of pregnancy-related complications, whether they know that they need treatment, whether health facilities are available and accessible, and also whether women receive adequate treatment at the health facilities (1). Every woman needs to be aware of the danger signs that occur during pregnancy, as complications can be unpredictable. These danger signs usually indicate the presence of an obstetric complication that may arise during pregnancy, delivery or post-delivery. Knowledge of these danger signs will help women to make the right decisions and take appropriate healthcare seeking actions (7). Lack of perceived benefit from ANC discourages women from seeking ANC, especially their late attendance for first antenatal services during their pregnancy or prevents them from returning for follow up care (8). In other to improve the knowledge and accessibility to ANC services, pregnant women need to be exposed to health information and communication on the significance of regular and early antenatal visits through a carefully planned health education programme.

Health education intervention is given a priority among basic components of primary health care (PHC) and significance of health education intervention has also been discussed by the subdivision of the AlmaAta Declaration of 1978 in that people have the right to know about the prevailing health problems occurred to them and to participate in practices for preventing and controlling them (9). On the patient bill of right, it was stressed that a client/patient has the right to access accurate and easily understood information respecting his/her health plan, hence, clients/patients have to be included when deciding the type of treatment to be given to him/her through health education in order to guide them to choose the treatment type by the health care professionals at health facilities (10). Studies conducted regarding the impact of health education programme on family planning knowledge, attitudes, and practices of married women in an army barrack in Nigeria as well as its effect on awareness of and attitude toward organ donation and transplantation of students of Maiduguri University showed that health education programme is an effective intervention for improving knowledge about and attitudes towards contraceptives use among married women in military barracks in Kaduna as well as willingness of students to donate organs for transplant to the recipients in Maiduguri $(11,12)$.

Many pregnant women in Edu local government area (LGA) attended their first ANC visit very late and refused follow-up ANC and subsequently delivered their babies at home with the help of unskilled attendants in an unhygienic condition. Delay in attending ANC visit put pregnant women of Edu LGA at high risks of pregnancy associated problems and consequently led to the loss of lives among mothers and newborn babies during or shortly after delivery of baby due to unidentified pregnancy complications. The prevalence of maternal dead was on the increase in Edu LGA, between January and December 2015, 15 pregnant women lost their lives during and shortly after delivery of baby and these losses of lives have become worrisome to not only the researchers but also the authorities of LGA (13). According to the data obtained on the observed maternal and child mortality and morbidity among pregnant women and poor attitudes of women towards accessing ANC services in Edu LGA, this study examined the impact of Health Education Intervention Programme (HEIP) on pregnant women's knowledge of and their accessibility to ANC services before and after intervention, in Edu LGA of Kwara State, Nigeria.

Accordingly, the following research questions were addressed to provide answers pertinent to the research problems:

1. What is the knowledge level of pregnant women regarding ANC services (ANCS) before and after HEIP in Edu LGA of Kwara State?

2. Will health education intervention programme have an impact on pregnant women's willingness to access ANC services in Edu LGA of Kwara State?

The hypotheses of the study indicate that health education intervention programme has no significant impact on knowledge and willingness of pregnant women to access ANC services before and after intervention programme in Edu LGA of Kwara State.

\section{Materials and Methods}

The present study which is a quasi-experimental research, using a pre- and post-test design, aimed at comparing pregnant women who exposed to health education intervention programme in experimental group with their counterparts in control group without being exposed to the same treatment in order to examine the impact of health education intervention on knowledge and accessibility of ANC services among women in Edu LGA of Kwara State. The population of the study consisted of pregnant women drawn from the local government total population of 201642 out of which 97602 were females (14). The LGA has three traditional districts with 10 political wards and 65 health facilities located in all communities of the LGA. Eleven out of 65 health facilities provide ANC services among others. The sample population for this study comprises of 120 pregnant women who were either pregnant or had one or more children selected from among 160 households in the two districts of Edu LGA.

Multiple sampling methods were applied in this study. First, simple random sampling technique was used to select 6 political wards ( 3 wards from Lafiagi \& 3 wards from Tsaragi district were included in experimental and control groups, respectively). Next, households 
were selected from each of the political wards using a systematic sampling procedure; that is, researchers and research assistants conducted a house numbering in each ward and the houses were chosen from the buildings list in each ward at specified intervals of every third building from a starting number depending on the length of the street. In each of the household selected, there were two or more pregnant women who were pregnant or had one child or more. Then, purposive sampling method was used to select 120 pregnant women out of 160 households in the two studied districts in the LGA.

The instrument applied in this study was a 25 -item tailormade questionnaire which elicited women's knowledge regarding ANC services and their access to such services. The questionnaire consists of two sections; Section A, that seeks information on pregnant women's knowledge regarding ANC services in the form of Yes/No questions mode and Section B, which was a 3-point response of modified Likert-type scale used to obtain information regarding accessibility of ANC service by pregnant women in Edu LGA of Kwara State. The following values were assigned to responses provided for Likert scale items: A - Always (5 points), ST - Some Times (3 points), and NA - Not Always (1 point). The instrument covers all the components of ANC services to ensure face and content validity upon which pregnant women's knowledge and accessibility to ANC service were defined. The items of the questionnaire were translated into Nupe language (a Volta-Niger language of the Nupoid branch spoken by the Nupe people of the Middle Belt region of Nigeria) and used as the interview schedule for pregnant women who could neither read nor write in English. The reliability of the questionnaire was determined by pre-test using a split-half test of Cronbach $\alpha$. The Cronbach a coefficient showed a total estimation of 0.68 which was obtained from a pilot study conducted on the neighbouring communities of Patigi LGA who were similar to those of the main study in terms of characteristics.

\section{Data Collection Procedure}

The data was collected in 3 steps: a pre-intervention (pretest), intervention and post intervention (post-test).

Pre-intervention: The pre-intervention phase, involved visit-supportive community leaders and the head of the households as well as samples of women for the study. The research assistants were recruited to help in administering the question items to pregnant women as pre-test.

Pre-intervention activities: Health personnel which comprises of nurses and community health educators working in the health centers of the target communities were selected and trained as research assistants by the researchers. Three types of educational materials were used to communicate health messages that is, pamphlet and posters, docudrama (video documentation), and a maternal health post sign chart. The first week spent seeking permission and cooperation of visit-supportive community leaders in the three study areas to carry out the study.

The husbands and household heads of the pregnant women residents were visited in order to explain the purpose of the study. On the second week, a day and time was fixed for the meeting with the research assistants following the development of the instructional materials and a training workshop was conducted for research assistants. Another meeting was scheduled the second day of the week with the researchers and pregnant women who volunteered to participate in the study. The same pretested instrument using a semi-structured questionnaire was administered to I20 content pregnant women (60 women in experimental group and 60 women in control group, respectively). This interview schedule was used by the researchers and research assistants to obtain information from pregnant women in the communities on the pre-tests day.

Intervention phase: On the first day of the intervention, pregnant women were briefed separately on the purpose of the study and allowed to discuss freely asking questions for clarifications by the researchers. The pregnant women were classified into two groups based on their residential districts; one served as the experimental group (60 women from Lafiagi district) who exposed to health education intervention relating to ANCS and the other was the control group (60 women from Tsaragi district) who were given health education intervention regarding the transmission and control of HIV/AIDS. The classification was carried out on district basis which is about 160 kilometers apart from control exchange of information among women in the two groups. The degree of separation of women in the experimental and control groups might not be perfect because the districts share common boundaries and this made it impossible to randomize the subjects in the groups. However, the decision on which district to serve as the experimental or control group was determined by the maternal health care services' accessibility outcome as revealed by the health information unit of the LGA.

The community-based health education intervention was conducted for the period of ten weeks that is, from the second week to the eleventh week of the study. The HEIP started with a video show and health teaching regarding ANCS on experimental group and health teaching respecting transmission and control of HIV/ AIDS on control group every Thursdays and Sundays at the village primary school and Town halls designated for the intervention throughout the intervention sessions. The researchers met experimental and control groups twice a week for 45 minutes of health education intervention programme per month at community level on a ten-week basis using lecture and discussion methods of health education. At the end of the ten-week health education intervention programme, a revision week of all the topics taught was conducted on the eleventh week on both experimental and control groups respectively. 
Post-intervention phase: The post-intervention session was conducted on the twelfth week of the intervention phase. The researchers and research assistants readministered the same sets of questionnaire/interview schedule used during the pre-test phase to pregnant women in both experimental and control groups at community levels in the two districts.

\section{Data Analysis}

Descriptive statistics (mean and standard deviation) for each item of the questionnaire were used to answer research questions. Besides, since it was assumed that data distribution was normal and that the study used a quasi-experimental research design for pre- and post-interventions, inferential statistic of paired $t$ test was applied to test the null hypotheses at 0.05 level of significance; it was used as a criterion for either rejecting or confirming the null hypotheses. The points scored by respondents on knowledge as well as accessibility were summarized using percentage mean score from their responses and thus: sum of scores on items

$$
3 \times \text { No. of items } \times 100 \%
$$

Knowledge of the respondents on the items relating to ANCS were divided into two categories: "high knowledge" for respondents who chose "Yes" and "low knowledge" for the respondent that chose "No". On the other hand, accessibility was classified into three categories: "very high accessibility" if the respondents chose "Always", "high accessibility" in case the respondent chose "Not always", and "poor accessibility" if the respondents chose "Not at all” option.

\section{Results}

Research Question 1

The first research question was:

What is the knowledge level of pregnant women regarding ANC service before and after health education intervention programme in Edu LGA of Kwara State?

Table 1 shows the pre-intervention mean scores (percentage) of 81.17 and 77.67 for experimental and control groups, respectively, while post-intervention demonstrates the mean score of 91.66 (percentage) for experimental group as well as the mean score of 76.33 (percentage) for control group. This result showed high level of knowledge about ANCS by pregnant women before intervention in both experimental and control groups; this is probably due to community support or routine maternal health services programmes provided in the communities of Edu LGA before the intervention programme. The result also displayed a very high level of pregnant women' knowledge in experimental group with regard to ANC services after health education intervention programme as compared to those in control group.

As regards the first research question, the following null-hypothesis was proposed:

Hypothesis 1: Health education intervention programme has no significant impact on the knowledge of pregnant women regarding ANC services in Edu LGA of Kwara State.

As can be seen in Table 2, pre- and post-intervention means for experimental group were 81.67 and 91.67 respectively, with a $\mathrm{t}$ value of 3.73 greater than table value of 1.96; accordingly, this finding rejected null hypothesis at 0.05 level of significance. This implies that health education intervention respecting ANC services had positive impact on pregnant women's knowledge regarding ANCS for the experimental group.

\section{Research Question 2:}

The second research question was:

Will health education intervention programme have an impact on pregnant women's willingness to access ANC services in Edu LGA of Kwara State?

Table 3 shows the pre-intervention mean scores of 71.08 and 73.25 for the experimental and control groups, respectively, while post-intervention demonstrates mean scores of 93.33 for the experimental group and 68.58 for the control group. This implies that pregnant women demonstrate very high willingness to access antenatal care services in both experimental and control groups before intervention; however, health education intervention had improved the willingness of pregnant women to access ANCS among the experimental group as compared to control group after HEIP.

Table 1. Summary of Mean and Standard Deviation Scores (Percentage) on Knowledge of Pregnant Women Regarding Antenatal Care Services

\begin{tabular}{lcccc}
\hline \multirow{2}{*}{ Group } & & \multicolumn{2}{c}{ Pre-intervention } & \multicolumn{2}{c}{ Post -intervention } \\
\cline { 2 - 5 } & N & Mean \pm SD & SE & Mean \pm SD \\
Experimental group & 60 & $\mathbf{8 1 . 1 7} \pm \mathbf{1 7 . 6 7}$ & 2.28 & $91.66 \pm 12.77$ \\
Control group & 60 & $77.67 \pm 14.10$ & 1.82 & $76.33 \pm 21.46$ \\
\hline
\end{tabular}

Table 2. Summary of $t$ test on Knowledge About ANC Services Among Pregnant Women in Experimental Group

\begin{tabular}{lcccccc}
\hline Experimental Group, $\mathbf{n}=\mathbf{6 0}$ & Mean & SD & SE & df & $\boldsymbol{t}$ & $\boldsymbol{P}$ Value \\
\hline Pre-intervention & 81.67 & 17.67 & 2.28 & \multirow{2}{*}{59} & \multirow{2}{*}{3.73} & \multirow{2}{*}{0.028} \\
Post-intervention & 91.67 & 12.78 & 1.64 & & & \\
\hline
\end{tabular}

$t=3.73, d f=58, P<0.05$ 
Table 3. Summary of Mean and Standard Deviation Scores on Accessibility to Antenatal Care Services Among Pregnant Women

\begin{tabular}{|c|c|c|c|c|}
\hline \multirow{2}{*}{ Group } & \multicolumn{2}{|c|}{ Pre-intervention: $n_{1}=60$} & \multicolumn{2}{|c|}{ Post- intervention: $n_{2}=60$} \\
\hline & Mean $\pm S . D$ & Standard Error Mean & Mean \pm SD & Standard Error Mean \\
\hline Experimental group & $71.08 \pm 37.93$ & 4.90 & $93.33 \pm 22.03$ & 2.84 \\
\hline Control group & $73.25 \pm 34.74$ & 4.48 & $68.58 \pm 37.04$ & 4.78 \\
\hline
\end{tabular}

Table 4. Summary of $T$ Test Regarding Accessibility of Pregnant Women to Antenatal Care Services

\begin{tabular}{lccccc}
\hline Experimental Group, $\mathrm{n}=\mathbf{6 0}$ & Mean & SD & SE & $\boldsymbol{d f}$ & $\boldsymbol{t}$ \\
\hline Pre-intervention & 78.92 & 32.17 & 4.88 & 59 & 3.00 \\
Post-intervention & 93.25 & 18.34 & 2.36 & & \\
\hline
\end{tabular}

$t=3.00, d f=59, P<0.05$

As regards the second research question, the following null-hypothesis was proposed:

Hypothesis 2: Health education intervention programme has no significant impact on the willingness of pregnant women to access antenatal care services in health centers in Edu LGA.

Table 4 represents pre-intervention mean of 78.92 and post intervention mean of 93.25 with a $t$ value of 3.00 . The $\mathrm{t}$-calculated (3.00) $>\mathrm{t}$ - tabulated (1.96), accordingly, the null hypothesis is rejected at 0.05 level of significance. This implied that health education intervention had a significant impact on pregnant women's willingness to access ANCS.

\section{Discussion}

The results of the study indicated that health education intervention programme had an impact on knowledge and accessibility of pregnant women regarding ANCS. In addition, the results revealed high level percentage of mean scores on knowledge of pregnant women with regard to ANCS before HEIP indicating high baseline knowledge of women in Edu LGA. This high knowledge level of pregnant women before HEIP might probably be due to various sources of health information relating to knowledge, especially during their pregnancy and their need to visit antenatal clinic for ANCS. These women acknowledged the importance of ANCS and admitted that such services provided the opportunity for them and their unborn babies to receive routine immunization, routine drugs, and other ANCS which give protection against various communicable diseases. This implied that in Edu LGA, pregnant women have adequate knowledge about ANC and are aware of when to access such services before intervention. This finding supported a study which posited that goals of antenatal care are to prevent health problems to both mother and child and that extended antenatal care is a significant period for pregnant women in area of interventions, information and fostering of health, wellbeing as well as survival of women and infants. Antennal care visit on the part of the women is an opportunity for these women and their health care givers to provide a delivery plan based on their unique needs, resources, and circumstances. The delivery plan identifies women's intentions about where and with whom they intend to give birth and contingency plans in the event of complications (transport, place of referral, etc) (11).

The pregnant women's knowledge baseline about ANCS probably influences their willingness to access various maternal health services especially ANCS. This finding confirms the study on the knowledge and accessibility to ANCS among pregnant women in Ilorin. The study observed that more than two thirds of the respondents (87.7\%) were aware of ANCS before the intervention (15). The finding of the study was also in line with the attitudes of some scholars who believed that knowledge is a crucial precondition for determining the behavior and attendance of women in antenatal clinic in Shanghai, China where women who attended ANC demonstrated a high degree of knowledge respecting the importance of attending ANC and breastfeeding of babies $(6,15)$. The studies further found that greater numbers of pregnant women were very knowledgeable about child immunization (95.8\%). Although $71 \%$ agreed that antenatal care was necessary, more than half of the women did not know that the first gestational month determines their first antenatal visit (16).

This study revealed pregnant women's satisfaction over the health education package delivered during their ANC visits and acknowledged the importance of maternal health services in prevention of pregnancy associated problems and death of mothers during delivery. This finding also corroborates with This finding agreed with the finding of a study in Ilorin, which concluded that there was high level of awareness of antenatal care services among women who were exposed to health teaching. And that majority of women had good knowledge on the activities carried out during the antenatal care demonstrated by high attendance at the health center (17). This implies that HEIP had a positive impact on the knowledge of pregnant women regarding ANCS. In addition, these findings supported the opinions of several researchers respecting the fact that knowledge about the importance of health services has the potential to positively shape health-seeking behaviour in pregnant women $(4,8,15,18,19)$. 
Health education intervention programme in this study had a positive impact on accessibility of pregnant women to ANCS in Edu LGA, Kwara State, Nigeria. The increased mean scores relating to willingness to access ANCS were in the areas of visiting health centers for health talks on good nutrition and exclusive breast feeding during ANC visits. Such findings are consistent with previous study which claimed that there was a high rate of antenatal care attendance among the respondents (76.8\%) when they had their last pregnancy (13). The finding of this study also corroborates a similar study which observed that health education has a positive effect on the knowledge of women, relating to breast self-examination (BSE), and students exposed to HEIP regarding organ donation $(6,12,20)$. Their studies showed a high significant improvement on the frequency and appropriate time to seek medical attention, an improvement shift from pretest to post-test. Conversely, however, the findings of this study do not match with the study on ignorance, lack of funds push up maternal death which opined that lack of knowledge and funds are factors causing maternal death in Indonesia. This is due to the fact that pregnant women, because of their ignorance about ANCS, do not receive an appropriate antenatal care crucial to their health (21). Based on the findings of this study, it was convincingly clear that health education intervention programme using ANCS teaching protocol had a positive impact on the knowledge of pregnant women relating to ANCS in Edu LGA.

Unfortunately, pregnant women's willingness to access some ANCS such as reporting obstetric care services and completion of routine immunization, recorded low mean score; this is probably a reflection of pregnant women low level of knowledge regarding the significance of immunization in relation to obstetric care services before intervention. However, the post-intervention session showed high mean scores of pregnant women's willingness to access ANCS in the areas of attendance in abdomen examination and weighing, screening of blood as well as urine testing sessions. The pregnant women's participation on health education sessions during ANC visits has significantly improved and that it provided pregnant women with opportunity to report for obstetric care services at the health centers in Edu LGA. The pregnant women improved willingness to access ANCS; it offered them the chances to complete their immunizations schedule which provides protection against peuperium sepsis, infection. This finding disagrees with demographic health survey reports of West African countries including Cameroun which shows a low level of accessibility of maternal care among women particularly in Nigeria compared with other African Countries. The survey reports further explained the accessibility distributions by countries; in Nigerian about $60.3 \%$ of mothers accessed ANCS during their last child birth, the comparative figures were $88.0 \%$ for Benin Republic (DHS, 2006), 72.8\% for
Burkina Faso (DHS, 2007), 83.4\% for Cameroon (DHS, 2004), and $91.9 \%$ for Ghana (DHS, 2003), respectively (22).

In conclusion, it is pertinent to note that health education intervention programme has a significant impact on both knowledge and access to ANCS among pregnant women in Edu LGA. To sustain knowledge gained during intervention sessions and to improve willingness of women to access ANCS, pregnant women's health seeking behaviours in communities of Edu LGA has to be supported in order to reduce maternal as well as child morbidity and mortality rate among women and children in Edu LGA of Kwara State, Nigeria. Based on this conclusion, it was recommended that governments at all levels of health care especially in Edu LGA PHC levels, strengthen the existing knowledge level of pregnant women regarding ANCS at communities' level. Maternal health services as a matter of policy should be instituted in all health institutions in the LGAs and State with more emphasis on health education intervention among other PHC programmes. This is to improve and sustain knowledge gained by pregnant women during HEIP about ANCS in the communities of Edu LGA, Kwara State. Health education intervention programmes especially on ANCS should include faith-based interventions relating to awareness of pregnant women with regard to the importance of access to ANCS in Edu LGA.

\section{Conflict of Interests}

Authors declare that they have no conflict of interests.

\section{Ethical Issues}

To conduct this study, approval was obtained from the authorities of Edu LGA and Heads of the communities of the study areas. A permission letter was read to pregnant women for consent within the households selected for the study.

\section{Financial Support}

This study was self-financed by the authors.

\section{References}

1. AbouZahr C, Wardlaw T. Maternal Mortality in 2000: estimates by WHO, UNICEF and UNFPA. Geneva: WHO; 2004.

2. Di Mario S, Basevi V, Gori G, Spettoli D. What is the effectiveness of antenatal care? (Supplement), WHO Regional Office for Europe (Health Evidence Network report) 2005. Available from: http://www.euro.who.int/_ data/assets/pdf_file/0005/74660/E87997.pdf.

3. World Health Organization. Maternal mortality: estimates developed by WHO, UNICEF, UNFPA, and the World Bank. Geneva: WHO; 2007.

4. Federal Ministry of Health (FMOH: Nigeria). Road map for accelerating the attainment of the millennium development goals related to maternal and newborn health in Nigeria. Abuja, FMOH: 2005.

5. United Nations. The Millennium Development Goals 
Report 2011. New York: United Nations; 2011.

6. Salma K. Evidence-Based Cancer prevention: strategies for NGOs - A UICC handbook for Europe. https://www.uicc. org/evidence-based-cancer-prevention-strategies-ngosuicc-handbook-europe. Published 12 February 2005.

7. Pembe AB, Urassa DP, Carlstedt A, Lindmark G, Nyström L, Darj E. Rural Tanzanian women's awareness of danger signs of obstetric complications. BMC Pregnancy Childbirth. 2009;9:12. doi: 10.1186/1471-2393-9-12.

8. Myer L, Harrison A. Why do women seek antenatal care late? Perspectives from rural South Africa. J Midwifery Womens Health. 2003;48(4):268-272.

9. Biswas R. Maternal Care in India Reveals Gaps Between Urban and Rural, Rich and Poor. http://www. prb.org/Publications/Articles/2003/MaternalCare inIndiaRevealsGapsBetweenUrbanandRuralRichandPoor. aspx. Accessed 24 February, 2016.

10. American Hospital Association. Patient bill of right; 2010. http://www.qcc.cuny.edu/SocialSciences/ppecorino/ MEDICAL_ETHICS_TEXT/Chapter_6_Patient_Rights/ Readings_The\%20Patient_Bill_of_Rights.htm. Accessed 11 January 2017.

11. Abdulrazaq AG, Kabir S, Mohammad NS, Suleiman IH. The effect of educational intervention on family planning knowledge, attitudes, and practices among married women in a military barrack in northern Nigeria. Afr J Reprod Health. 2014;18(1):93-101.

12. Sanusi AA. Impact of Health Education Intervention Programme on awareness of and attitude to organ transplantation among University of Maiduguri students. A thesis project (unpublished); 2002. pp. 73-75.

13. Edu Local Government Area Health Information Unit. Annual records of maternal morbidity and mortality rate [unpublished].2015.

14. Nigeria: Administrative division (State and Local Government Areas)-Population Statistics, Charts and Map - City Population. CITY POPULATION website. 2006.
http://www.citypopulation.de/Nigeria.html.

15. Adewoye KR, Musa IO, Atoyebi OA, Babatunde OA. Knowledge and utilization of antenatal care services women of childbearing age in Ilorin-east Local Government Area, 2014. International Journal of Science and Technology. 2013;3(3):188-193.

16. Zhao Q, Kulane A, Gao Y, Xu B. Knowledge and attitude on maternal health care among rural-to-urban migrant women in Shanghai, China. BMC Womens Health. 2009;9:5. doi:10.1186/1472-6874-9-5

17. Adewoye KR, Musa IO, Atoyebi AO, Babatunde AO. Knowledge and utilization of antenatal care services women of childbearing age in Ilorin-east Local Government Area, 2014. International Journal of Science and Technology. 3(3):188-193.

18. Amoran OE. Impact of health education intervention on malaria prevention practices among nursing mothers in rural communities in Nigeria. Niger Med J. 2013;54(2):115122. doi:10.4103/0300-1652.110046

19. Smith K, Dmytraczenko T, Mensah B, Sidibe O. Knowledge, Attitudes, and Practices Related to Maternal Health in Bla, Mali: Results of a Baseline Survey. Maryland: Partners for Health Reform plus; 2004.

20. Abd El Aziz HM, Akl OA, Ibrahim HK. Impact of A Health Education Intervention Program about Breast Cancer among Women in a Semi-urban Area in Alexandria, Egypt. J Egypt Public Health Assoc. 2009;84(1-2):219-243.

21. DursinRichel. Ignorance, lack of funds push up maternal death. http://www.hartford-hwp.com/archieves/54b/074. html. Accessed 10 March 2011.

22. Magadi MA, Agwanda AO, Obare FO. A comparative analysis of the use of maternal health services between teenagers and older mothers in sub-Saharan Africa: evidence from Demographic and Health Surveys (DHS). Soc Sci Med. 2007;64(6):1311-1325. doi:10.1016/j. socscimed.2006.11.004

(C) 2018 The Author (s); This is an open-access article distributed under the terms of the Creative Commons Attribution License (http://creativecommons.org/licenses/by/4.0), which permits unrestricted use, distribution, and reproduction in any medium, provided the original work is properly cited. 